\title{
Magnetism and Metal-Insulator Transition in III-V Based Diluted Magnetic Semiconductors
}

\author{
S. Katsumoto ${ }^{1,2}$, T. Hayashi ${ }^{1}$, Y. Hashimoto ${ }^{1}$, Y. Iye $^{1,2}$ \\ Y. Ishiwata ${ }^{1}$, M. Watanabe ${ }^{3}$, R. Eguchi ${ }^{1}$, T. Takeuchi ${ }^{4}$, Y. Harada ${ }^{3}$, S. Shin $^{1,3}$ \\ and K. Hirakawa ${ }^{2,5}$ \\ ${ }^{1}$ Institute for Solid State Physics, University of Tokyo, \\ 5-1-5 Kashiwanoha, Kashiwa, Chiba 277-8581, Japan \\ ${ }^{2}$ CREST, Japan Science and Technology Corporation, \\ Mejiro, Tokyo 171-0031, Japan \\ ${ }^{3}$ RIKEN, Sayo-gun, Hyogo 679-5143, Japan \\ ${ }^{4}$ Department of Applied Physics, Science University of Tokyo \\ Kagurazaka, Shinjuku-ku, Tokyo 162-8601, Japan \\ ${ }^{5}$ Institute of Industrial Science, University of Tokyo, \\ 7-22-1 Roppongi, Minato-ku, Tokyo 106-8558, Japan
}

\begin{abstract}
We report experiments on the magnetism and the transport in III-V based diluted magnetic semiconductors (Ga,Mn)As and (In,Mn)As. Heat treatment (annealing) at comparatively low temperatures (slightly above the growth temperature) is unexpectedly found to be effective to improve the ferromagnetism and the metallic conduction. Infrared optical conductivity measurements and soft x-ray absorption spectroscopy reveal that the double exchange model is convenient to describe the ferromagnetism. The transport in the vicinity of metal-insulator critical point was studied in detail by using the low-temperature annealing method.
\end{abstract}

\section{$\S 1 \quad$ Introduction}

Diluted magnetic semiconductors (DMS's) synthesized by introduction of magnetic ions into semiconductors are now expanding a new horizon of "spin-electronics". Especially III-V based DMS's (In,Mn)As and (Ga,Mn)As have attracted much attention. They undergo ferromagnetic transition at comparatively high temperatures and have high connectivity to sophisticated III-V artificial superstructures. A remarkable feature of the ferromagnetism is that it is mediated by the doped holes, which fact was demonstrated by illumination-driven ferromagnetic transition $^{3)}$ and by disappearance of the ferromagnetism by counter doping ${ }^{4}$.

However the physical mechanism of the ferromagnetism is still under discussion. In a II-IV DMS, it is claimed that the Ruderman-Kittel-Kasuya-Yosida (RKKY) interaction is the origin of the transient ferromagnetism ${ }^{5)}$. The transport and the response to external magnetic field in (Ga,Mn)As were analyzed along similar interpretation ${ }^{6}$. This interpretation, however, has an essential difficulty that the estimated amplitude of the exchange constant tends to exceed the Fermi energy. While interpretations to solve this inconsistency were attempted ${ }^{7}$, a calculation of band structure $\left.{ }^{8}\right)$ in (In,Mn)As suggested doubleexchange (DE) mechanism is more plausible. Though the result of the band-calculation is also getting experimental supports $^{9)}$, decisive evidence has not appeared yet. In a sense, these two pictures describe limiting cases and the reality should exist between them. It would be meaningful, however, to examine which picture is convenient to describe the experiments.

These materials also shows exotic properties in transport ${ }^{10)}$. Increasing $\mathrm{Mn}$ concentration $(x)$ from the dilute limit, they undergo an insulator-to-metal transition (the first MIT). The ferromagnetism appears at a Mn concentration slightly lower than the first MI critical point. Further increase in $x$ causes a metal-to-insulator re-entrant transition (the second MIT).

One of the problems in these materials for researches and applications is that the qualities of the grown crystals are too sensitive to the growth conditions because the conditions are very far from equilibrium ${ }^{11)}$. In this article, we report that annealing (heat treatment) at comparatively low temperatures partly solve the problem. We apply this method to explore the picture for the ferromagnetism, and the nature of the second MIT. Owing to limited space, we mainly discuss the case of (Ga,Mn)As.

\section{$\S 2$ Experiment}

(Ga,Mn)As films were grown by low temperature molecular beam epitaxy (LT-MBE) onto semi-insulating (001) GaAs substrates. The growth temperature was around $230 \mathrm{C}$, and the V/III ratio was about 5 . These parameters were optimized so as to obtain highest $T_{\mathrm{C}}$ for each Mn concentration. The nominal Mn concentrations were determined from (004) diffraction of x-ray in the as-grown 
samples.

The effect of low-temperature annealing was examined mainly at $x=0.05$. The as-grown wafer was cleaved into squares of $4 \times 4 \mathrm{~mm}^{2}$. The annealing was performed in $\mathrm{N}_{2}$ gas flow without any coverage of the surfaces ${ }^{12)}$. The annealing time was fixed to 15 minutes.

The transport above $2 \mathrm{~K}$ was measured by a conventional AC bridge with van der Pauw method in a He circulation cryostat. Below 2K, we prepared samples with a Hall-bar shape by mesa-etching, which were cooled down to $50 \mathrm{mK}$ by a ${ }^{3} \mathrm{He}-{ }^{4} \mathrm{He}$ dilution refrigerator.

For infrared conductivity measurement, the wafers were cleaved into pieces of $5 \times 5 \mathrm{~mm}^{2}$ in a He gas flow cryostat. The conductivities were measured in a transmissiongeometry by using a Fourier transform spectrometer. The optical absorption coefficient $\alpha(\omega)$ of the epitaxial film (Ga,Mn)As is expressed by the transmission spectrum of the insulating GaAs substrate $t_{\mathrm{GaAs}}$ and that of the film $t_{\text {GaMnAs }}$ as

$$
\alpha(\omega)=\log \left(\frac{t_{\mathrm{GaAs}}}{t_{\mathrm{GaMnAs}}}\right) / d
$$

where $d$ is the thickness of the film. The transmission spectra are also connected to the optical conductivity $\sigma(\omega)$ through

$$
\frac{t_{\mathrm{GaAs}}}{t_{\mathrm{GaMnAs}}}=\left|1+\frac{\sigma(\omega) d}{Y_{0}+Y_{s}}\right|^{2},
$$

wher $Y_{0}$ and $Y_{s}$ are the admittances of the vacuum and GaAs, respectively.

The x-ray absorption spectroscopy (XAS) was performed by using a soft-x-ray spectrometer installed at the undulator beamline BL-2C in Photon Factory of KEK. Synchrotron radiation was monochromatized using a varied line spacing plane grating, whoes energy resolution was less than $0.1 \mathrm{eV}$ at $650 \mathrm{eV}$. The spectra were taken at room temperatures.

The growth conditions for (In,Mn)As were the same for ( $\mathrm{Ga}, \mathrm{Mn})$ As besides the growth temperature, which were 30 degrees higher than that for (Ga,Mn)As.

\section{$\S 3$ Effect of annealing on the transport and magnetism}

The Mn concentration dependence of the Curie temperature $\left(T_{\mathrm{C}}\right)$ in as-grown samples was similar to that reported previously ${ }^{10,13)}$ when the growth conditions are optimized to maximize $T_{\mathrm{C}}$. However slight difference in the conditions results in severe degradation in transport and magnetism especially around the second MIT. For example, 10 degree difference in the growth temperature makes the conduction from metallic to insulating even though the growth mode observed by refractive high energy electron diffraction (RHEED) does not change ${ }^{11)}$. This high sensitivity arises from the fact that the growth is very far from the equilibrium. This would be an obstacle for research and application of these materials. In the following we show that low-temperature annealing after the growth would be a key to solve this problem.

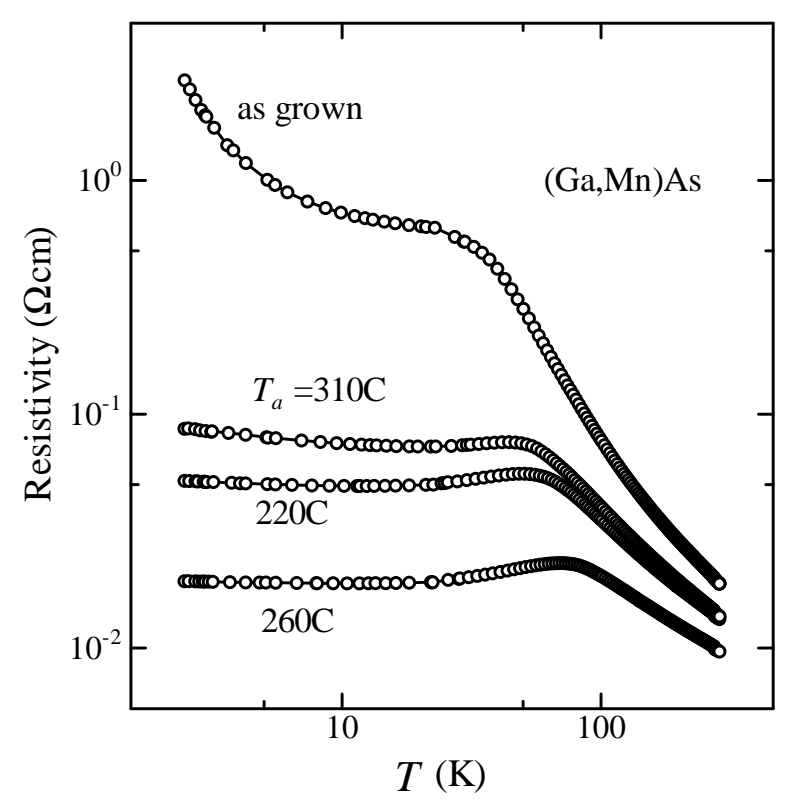

Figure 1: Temperature dependence of resistivity in as-grown and annealed (Ga,Mn)As films.

Figure 1 shows the temperature dependence of resistivity in $(\mathrm{Ga}, \mathrm{Mn})$ As with nominal Mn concentration of $5 \%$. The as-grown sample shows strongly insulating behavior with a hump structure around $30 \mathrm{~K}$. This hump is known to appear around $T_{\mathrm{C}}$. As demonstrated in Fig.1, by the annealing at comparatively low temperature (slightly above the growth temperature), the resistivity was lowered and the hump moved to higher temperatures. This is surprising because it was reported that the annealing at higher temperatures results in the clustering of $\mathrm{MnAs}^{14)}$ and even at comparatively low temperatures the annealing causes degradation in the transport and the magnetism ${ }^{15)}$.

We summarize the effect of the annealing in Fig.2. $T_{\mathrm{C}}$ and the hole concentration $p$, which was estimated from room temperature Hall coefficient, show maxima around the annealing temperature $\left(T_{a}\right)$ of $260^{\circ} \mathrm{C}$ while the lattice constant $a$ decreases monotonically with $T_{a}$. The decrease in $a$ is steeper for the higher $T_{a}$. For this decrease in $a$, it is hardly conceivable that $\mathrm{Mn}$ atoms ran away from the substrate considering the inherent vapor pressure. There was no increase in full width at half maximum of the $\mathrm{X}$ ray diffraction peak, which manifests that the annealing caused no significant inhomogeneity in the films. On the other hand, what is remarkable is that $T_{\mathrm{C}}$ reaches $95 \mathrm{~K}$, which is highest among so far reported for (Ga,Mn)As directly grown onto GaAs. A more important experimental fact, which cannot be seen in Fig.2, is that the highest $T_{\mathrm{C}}$ obtained by the annealing does not strongly depend on $T_{a}$. That is, even if $T_{a}$ is lower than $260^{\circ} \mathrm{C}$, longer annealing time enhances $T_{\mathrm{C}}$ to the highest value obtained for $T_{a}=260^{\circ} \mathrm{C}$ in the end. This means that by adopting the low-temperature annealing, we can overcome the difficulty due to the sensitivity of the crystalline quality to 
the growth parameters ${ }^{16)}$.

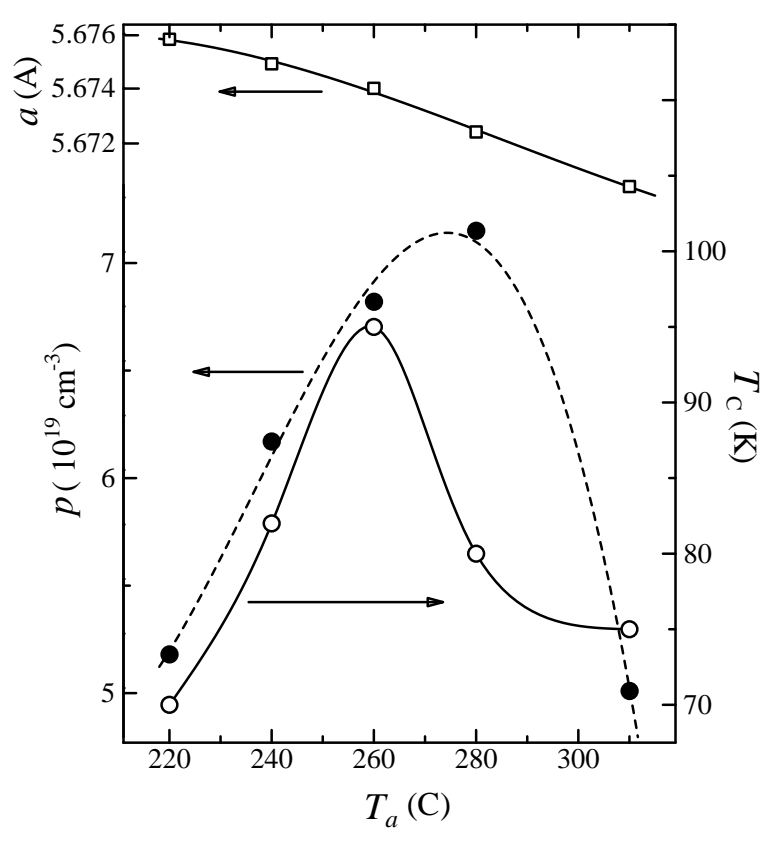

Figure 2: Material parameters in $\mathrm{Ga}_{0.95} \mathrm{Mn}_{0.05} \mathrm{As}$ (lattice constant $a$, Curie temperature $T_{\mathrm{C}}$, and hole concentration $p$ ) as a function of annealing temperature $T_{a}$.

A natural question is what happens in the annealing process. Because $x$ of $5 \%$ is close to the second MI transition, one might suspect that precipitation of MnAs occurs during annealing and the decrease in the effective Mn concentration caused the increase in $T_{\mathrm{C}}$ and the conductivity. However this is not only simply eliminated by the fact that $T_{\mathrm{C}}$ reaches higher value than those obtained in as-grown samples but also denied by the dependence of $T_{\mathrm{C}}{ }^{16)}$.

Hence answering the above question would be a key to clarify more essential issue, i.e., the mechanism of the ferromagnetism.

The annealing technique also gives us a means to control material parameters finely in a single wafer. We can thus obtain, e.g., a sample which is very close to the MI critical point. In the following sections we discuss our present understanding of these issues based on the experiments on infrared absorption and soft X-ray absorption.

\section{$\S 4$ Infrared absorption spectra}

Figure 3 shows optical conductivity spectrum $\sigma(\omega)$ of a metallic sample with $x=4.6 \%^{17)}$. The gap in the spectra is due to the Reststrahlen band of GaAs. $\sigma(\omega)$ is almost independent of $\omega$ at high temperatures, indicating very short scattering time of holes. At $5 \mathrm{~K}, \sigma(\omega)$ increases with increasing $\omega$. This behavior is suggestive of hopping transport rather than usual metallic Drude conduction.

It also suggests us to reconsider the "metallic" DC conduction in $\left.(\mathrm{Ga}, \mathrm{Mn}) \mathrm{As}^{10}\right)$. In the samples with $x$ in the range from $3.5 \%$ to $4.8 \%$, the temperature dependence of

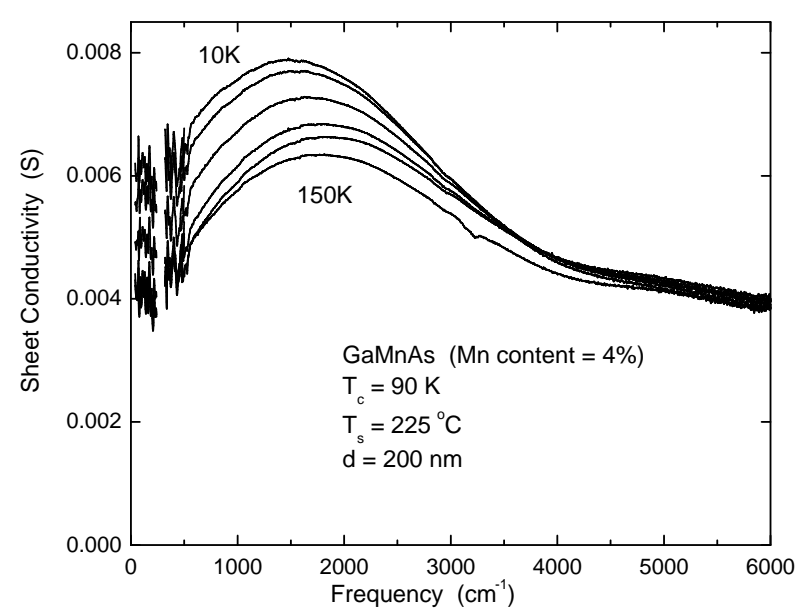

Figure 3: Optical conductivity spectrum $\sigma(\omega)$ of a metallic (Ga,Mn)As sample with $x=4.6 \%$. The parameter is temperature varying from down to top as $150,120,90,60,30,10$ $\mathrm{K}$.

the DC resistivity is obviously metallic. However the mean free path estimated from Hall mobility assuming simple Drude model, is around $10 \AA$, which is extremely short as heavily doped semiconductors. And this mean free path is, again according to the Drude theory, comparable with the Fermi wavelength, which result tells that the Drude picture is totally broken down. Instead of propagation of holes conserving momentum, we should consider conduction through wavefunctions with strong spatial modulation due to disorder. Hence in high energy region, the transport should be more or less hopping-like in accordance with $\sigma(\omega)$ spectra. It seems rather unnatural, then, to apply the picture of RKKY interaction, in which the basic assumptions are local moments and free electrons (holes) scattered by them.

In order to obtain further information, we investigated $\alpha(\omega)$ in higher frequency range. Figures 4 (a) and (b) show the $\alpha(\omega)$ spectra obtained for semiconducting and metallic samples respectively. The semiconducting one is as-grown while the metallic one is obtained by LT-annealing. While $\alpha(\omega)$ of the insulating and semiconducting samples vanish in the zero-frequency limit for low temperatures, that of the metallic sample remains finite for $\omega \rightarrow 0$, indicating the existence of delocalized holes. The most pronounced feature in these spectra is the broad conductivity peak at around $200 \mathrm{meV}$.

Besides the peak, a remarkable characteristic is the enhancement in $\alpha(\omega)$ with decreasing $T$ in the region below $400 \mathrm{meV}$. The enhancement sets in just at $T_{\mathrm{C}}$ and is most pronounced for the metallic sample. According to the sum rule in the oscillator strength, there should be some transfer of the spectral weight which corresponds to the enhancement. Such spectral transfer below $T_{\mathrm{C}}$ in a wide 
energy range is characteristic to DE-driven ferromagnets. Hence the observed transfer in the spectral weight of $\alpha(\omega)$ suggests that the double-exchange (together with $p-d$ exchange) is the dominant mechanism for the ferromagnetism.
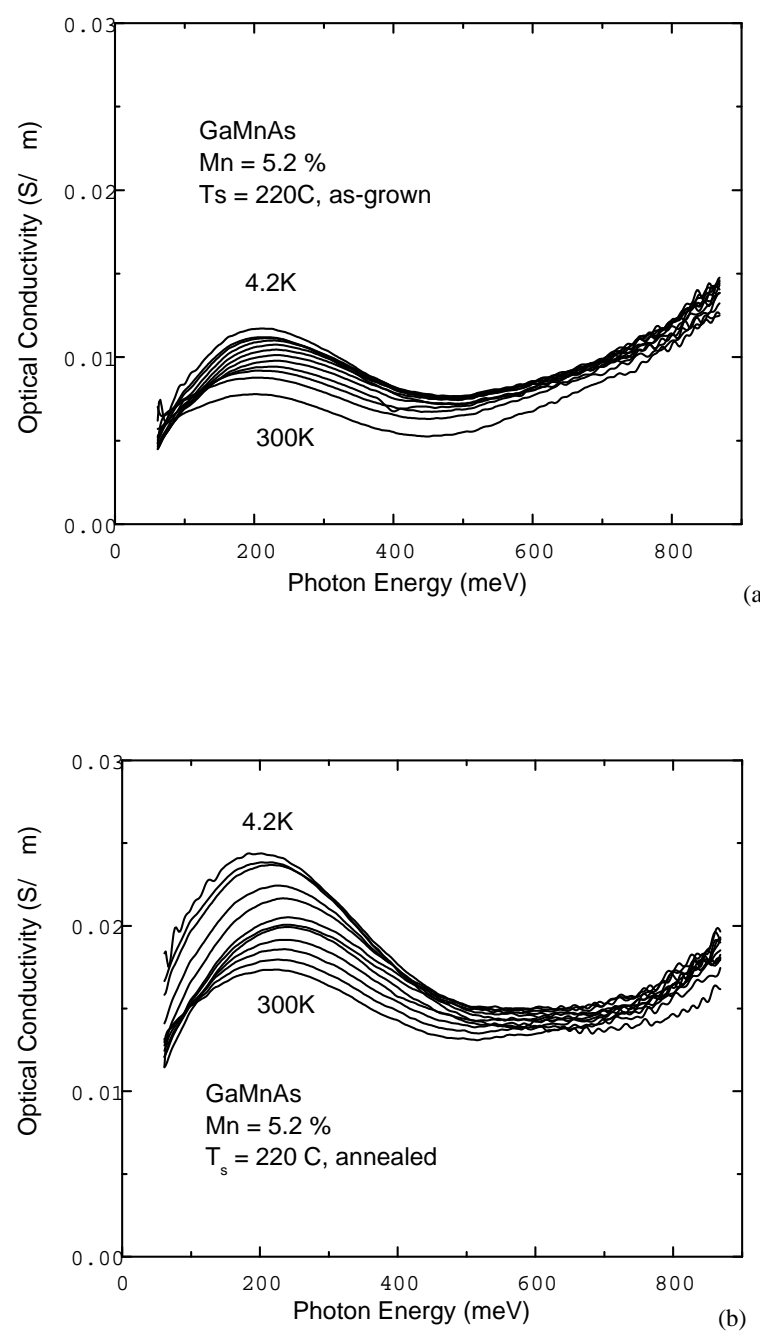

Figure 4: Absorption coefficient $\alpha(\omega)$ spectra obtained for semiconducting (a) and metallic (b) samples respectively. The semiconducting one is as-grown while the metallic one is obtained by LT-annealing. The temperature was varied from down to top as 300,250,200,150,120, 100, 80,60,40,20, 10 and $4.2 \mathrm{~K}$.

Also for the peak structure around $200 \mathrm{meV}$, an interpretation along the DE mechanism is possible. The lattice configuration around Mn atom must be distorted by the Jahn-Teller effect and a hopping of a hole between Mn sites is associated with annihilation and creation of small polarons. When considerable amount of holes are localized forming small polarons, $\alpha(\omega)$ is expected to have a broad peak at about $2 E_{\mathrm{sp}}$, where $E_{\mathrm{sp}}$ is the formation energy of a small polaron and estimated to be about $100 \mathrm{meV}$ from
Fig.4. On the other hand, the linewidth is predicted to be $4\left(E_{\mathrm{sp}} \hbar \omega_{\mathrm{ph}}\right)^{1 / 2}$ for $k_{\mathrm{B}} T<\hbar \omega_{\mathrm{ph}}$, where $\hbar \omega_{\mathrm{ph}}$ is the relevant phonon energy. Substituting $\hbar \omega_{\text {ph }}$ with the GaAs phonon energy near the Brillouin zone boundary $(\sim 30 \mathrm{meV})$, we obtain a line width of $200 \mathrm{meV}$, which reasonably agrees with the results in Fig.4.

The DE model has been said to describe several manganites such as ( $\mathrm{La}, \mathrm{Sr}) \mathrm{MnO}_{3}$, in which steep decrease of resistivity below $T_{\mathrm{C}}$ is observed. This is reminiscent of the resistivity humps shown in Fig.1. In previous reports, they are attributed to critical scattering of holes ${ }^{6,18)}$ at the transition temperature. However, as discussed above, the picture of "almost localized holes" seems to be more appropriate than such "scattering of free holes" picture to describe the present system. Especially the coherence of holes between scatterings by magnetic moments is essential for the critical scattering effect. It is thus natural to attribute the decrease of the resistivity below $T_{\mathrm{C}}$ to the delocalization of holes which gain the kinetic energy by the DE mechanism. The simplest DE model does not describe the temperature dependence above $T_{\mathrm{C}}$, though it is known that the combination of the DE Hamiltonian and electron-phonon coupling via the Jahn-Teller effect explains the "peak" feature around $T_{\mathrm{C}}{ }^{19)}$.

In the case of (In,Mn)As, clear Drude tailing to the low frequency limit appears in contrast to $(\mathrm{Ga}, \mathrm{Mn}) \mathrm{As}$, though "dirtiness" of the DC conduction is comparable. The transfer in the spectral weight is also much clearer indicating the double exchange picture works well. However, this transfer starts at a temperature much higher than $T_{\mathrm{C}}$. This transfer is probably related to the superparamagnetism, which appears at very high $(\sim 100 \mathrm{~K})$ temperatures.

\section{$\S 5$ Soft X-ray absorption spectra}

As seen in the previous section, it is more important to explore the local electronic structure around $\mathrm{Mn}$ ions in order to clarify the origin of the ferromagnetism. For this purpose, local probes of Mn atoms such as core level xray photoemission spectroscopy (XPS) ${ }^{9)}$ would be suitable. Here we report a study of local electronic structure in (Ga,Mn)As by using Mn $2 p$ x-ray absorption spectroscopy $(\mathrm{XAS})^{20)}$.

Figure 5(a) shows the XAS spectra of as-grown samples, while those of annealed samples are shown in Fig.5(b). The structures around $640 \mathrm{eV}$ and $651 \mathrm{eV}$ correspond to $L_{3}$ and $L_{2}$ levels respectively. The main peak $\left(L_{3}\right)$ in the as-grown samples has a doublet structure, which consists of peaks at $639.5 \mathrm{eV}$ and $640 \mathrm{eV}$. On the other hand in the annealed samples, only a single peak at $640 \mathrm{eV}$ appears for $L_{3}$ except in the sample with $x=0.032$. This "singlepeak" spectrum is very similar to a Mn $2 p$ XAS calculation based on atomic multiplet theory for $\mathrm{Mn}^{2+}\left(d^{5}\right)$ in tetrahedral $\left(T_{d}\right)$ co-ordination for the case with crystalfield strength $(10 D q)=0.5 \mathrm{eV}^{21)}$, manifesting $\mathrm{Mn}$ is in $T_{d}$ symmetry (Fig.6(a)).

This doublet $\rightarrow$ singlet transition by annealing indicates 


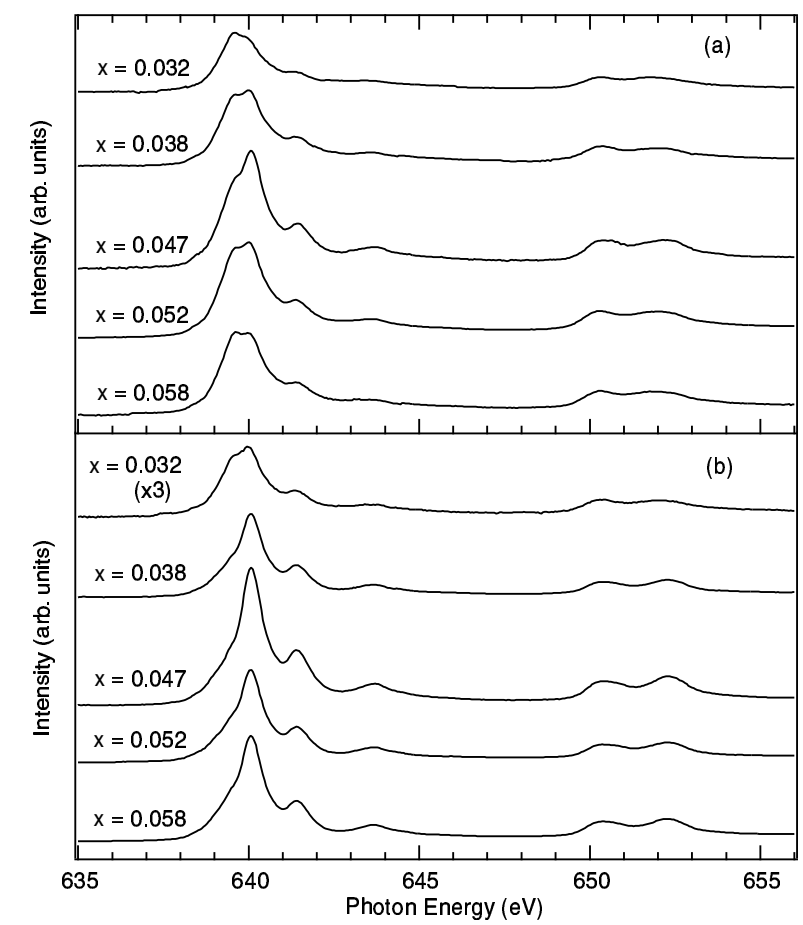

Figure 5: Mn $2 p$ XAS spectra for (a) as-grown and (b) LTannealed $(\mathrm{Ga}, \mathrm{Mn})$ As.

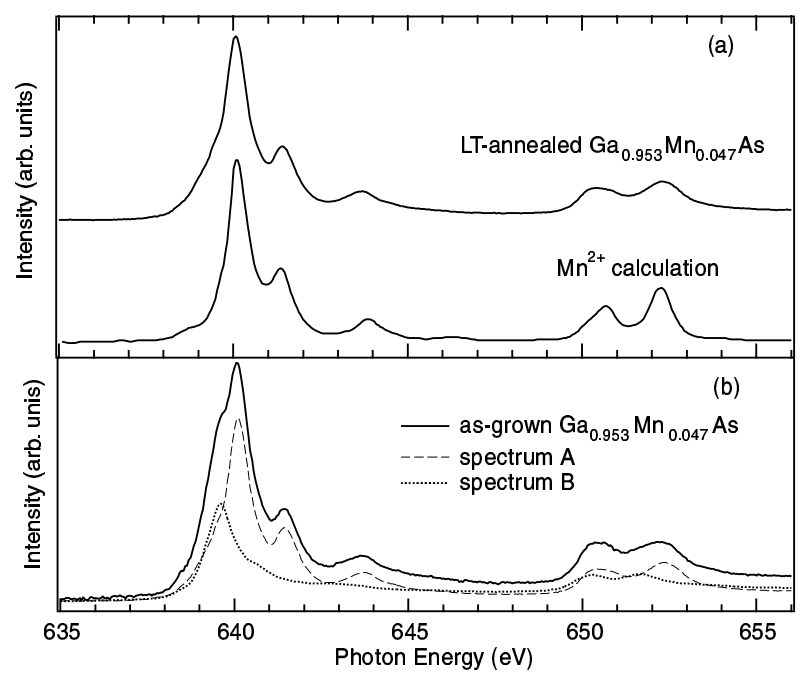

Figure 6: (a) Comparison between Mn $2 p$ XAS spectra for annealed GaMnAs with $x=4.7 \%$ and the calculation based on atomic multiplet theory for $\mathrm{Mn}^{2+}\left(d^{5}\right)$ in $T_{d}$ co-ordination for $10 D q=0.5 \mathrm{eV}^{21)}$. (b) An example of spectrum decomposed into two spectra. Solid line is the spectrum for as-grown sample with $x=4.7 \%$. The dashed and the dotted lines are spectrum $\mathrm{A}$ and $\mathrm{B}$ respectively. that two types of Mn ions exist in as-grown samples, one of which is transformed into the other type by annealing. Assuming that the spectrum for the annealed sample with $x=0.047$ is purely from one species, we decompose the other spectra into spectrum A and B (Fig.6(b)). Figure 7 displays intensities of these spectra and $T_{\mathrm{C}}$ as a function of $x$. It is apparent that $T_{\mathrm{C}}$ has close correlation with the intensity of spectrum A. Hence it is suggested that the species of Mn which produces the spectrum A generates the ferromagnetism.

The simplest interpretation of the annealing process, is disposal of excess As atoms through the evaporation from the surface. Such excess As atoms are known to work as deep donors and to compensate holes. Hence the annealing increases the concentration of $4 p$ holes. We tentatively assume that $\mathrm{Mn}$ which produce spectrum A are those normally replaced the Ga sites ${ }^{22)}$ and have four nearest neighbor As atoms. Since XAS gives site projected partial density of states, such a Mn is assigned to be Mn- $h$ (As $4 p$ hole) complex.

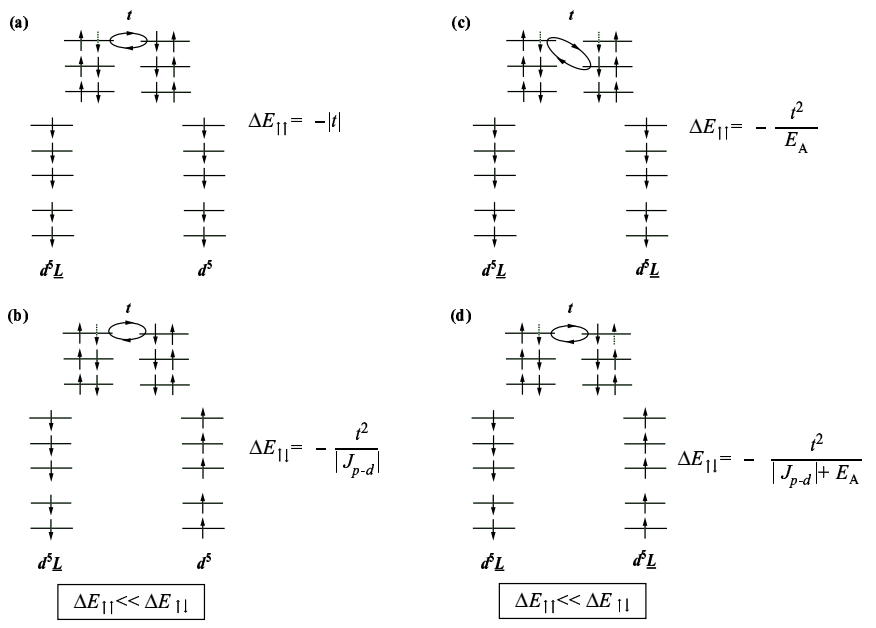

Figure 7: A possible energy level scheme of (Ga,Mn)As. Solid arrows stand for electrons while dotted arrows are for holes in As $4 p$ orbital. The diagrams are shown for the cases in which the magnetic moments of $\mathrm{Mn}-h$ complex and free $\mathrm{Mn}^{2+}$ ion are (a) parallel or (b) antiparallel, and two Mn- $h$ complexes are (c) parallel or (d) antiparallel. Here $J_{p-d}$ is assumed to be negative.

If we further assume that the Mn for spectrum B also have the same atomic configuration and the annealing only changes the compensation, the two kinds of Mn states are assigned to $d^{5} \underline{L}$ and $d^{5}$ configurations respectively for spectrum $\mathrm{A}$ and $\mathrm{B}$, where $\underline{L}$ represents a hole in the ligand As. The energy difference $(0.5 \mathrm{eV})$ between $d^{5} \underline{L}$ and $d^{5}$ estimated from the difference in the spectra can be understood from the electronic structure parameters obtained by Mn $2 p$ core-level photo-emission spectra ${ }^{9}$.

In the concluding part of these two sections, we discuss a possible mechanism for the ferromagnetism. Now it is clear that the holes almost localized around $\mathrm{Mn}^{2+}$ ions mediate the ferromagnetism. Possible schematic energy 
diagrams are shown in Fig.8, where the $p-d$ exchange interaction $\left(J_{p d}\right)$ is negative (anti-ferromagnetic). These diagrams explain that in order to gain the kinetic energy of $4 p$ holes, hopping between two Mn sites should be "allowed", which leads to the ferromagnetic order of $d$-spins through the $p-d$ hybridization $J_{p d}$. This is a kind of DE mechanism and naturally explains the present observations.

\section{$\S 6$ Metal-insulator transition}

Here we show an example of the application of the annealing technique to basic research. MI transition (MIT) is one of the central issues in solid state physics ${ }^{23)}$. The simplest mechanism of MI transition in doped semiconductors is the overlapping of donor (acceptor) wavefunctions and the formation of impurity bands. Actual MIT's are, however, considered to be dominated by other mechanisms especially in the vicinity of the critical points. Anderson localization is one of such mechanisms. Thus far, the MIT's in doped semiconductors have been considered in the context of Anderson localization plus Coulomb repulsion in the presence of disorder ${ }^{24)}$. On the other hand, in "strongly correlated" systems, Mott-type transition is often the central mechanism for the MIT's.

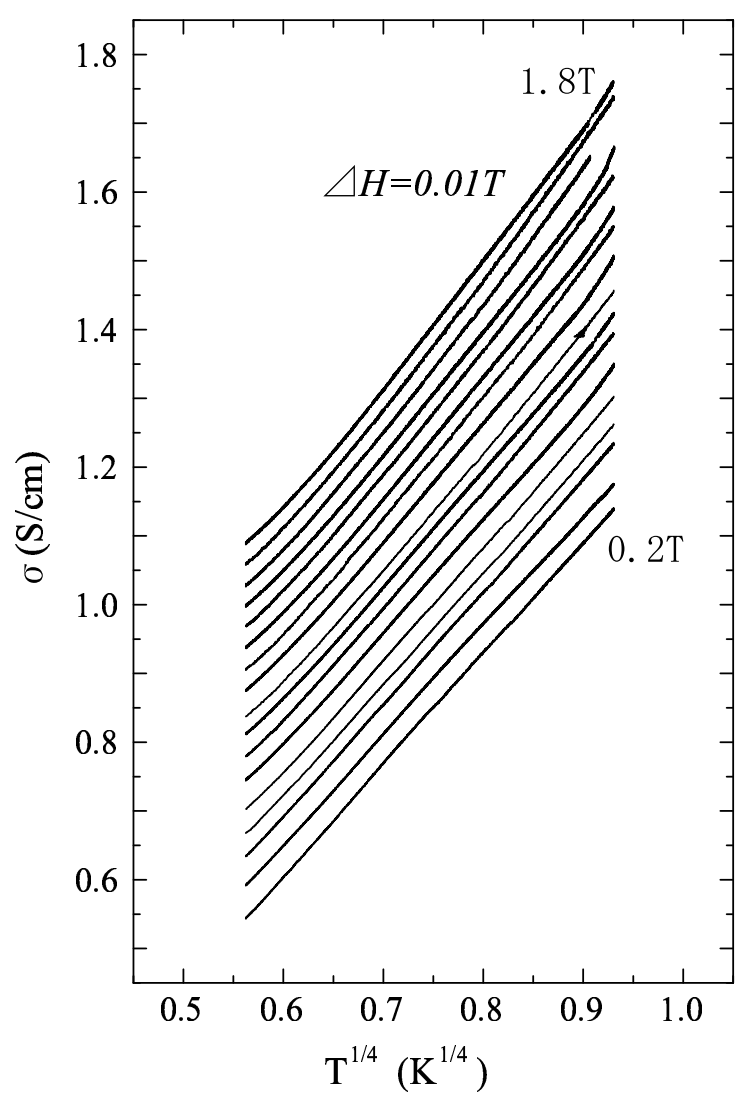

Figure 8: Temperature dependence of conductivity in the vicinity of the MIT critical point. The external magnetic field was varied from $0.2 \mathrm{~T}$ to $1.8 \mathrm{~T}$ with the step width of $0.01 \mathrm{~T}$. The abscissa is plotted as $T^{1 / 4}$.
The MIT's in (Ga,Mn)As and (In,Mn)As have unique characteristics. The critical Mn concentration is very far from the Mott's criterion ${ }^{23)}$, which manifests that these MIT's are so different from those of ordinal doped semiconductors. On the other hand, DE mechanism for ferromagnetism is associated with a MIT. However these materials undergo ferromagnetic transition even in the insulating phase. The disorder should thus play an important role in the MIT's. More remarkably, they showed the second MIT's when the Mn concentration is further increased. In these second MIT's, the disorder should be more important.

It is crucial to approach the critical points in the study of phase transitions though in the case of MIT's, the relevant parameter is often difficult to be varied in a single specimen. Here we tuned the resistivity of the (Ga,Mn)As sample $(x=0.05)$ by repeated short-time annealing at $260^{\circ} \mathrm{C}$ to just insulating side of the second MIT. Then by applying external magnetic field, the giant magnetoresitance ${ }^{10)}$ can bring the sample into the metallic side through the MIT critical point.

Figure 8 shows temperature dependence of the conductivity for various external magnetic field. The abscissa is plotted in $T^{1 / 4}$. The data points are in lines whose offsets at absolute zero cross zero-conductance indicating that $\sigma \propto T^{1 / 4}$ approximates the experiment around the critical point. The temperature dependence of $T^{1 / 2}$ is often observed at MIT's in compensated semiconductors. This means that in the present case, the temperature dependence $\mathrm{d} \sigma / \mathrm{d} T$ becomes steeper with lowering temperature compared with ordinal compensated semiconductors. This is qualitatively interpreted as follows. Just below $T_{\mathrm{C}}$, the ordering of $\mathrm{Mn}$ spins is favorable for conduction through the DE mechanism. However at lower temperatures the freezing of magnetic moments pointing random directions, would enhance effective degree of disorder and accelerate quenching of conduction.

Fortunately we can examine whether the conductance scales on $H$ (magnetic field) and $T$ consistently regardless of the detailed contents of the theoretical model through two-parameter scaling form ${ }^{24)}$;

$$
\sigma(H, T) \propto T^{x} f\left(\left|H / H_{c}-1\right| / T^{y}\right),
$$

where $f$ is a scaling function, $x$ and $y$ are parameters, giving the critical exponent $\nu=x / y$, which is defined by the critical behavior as

$$
\sigma(H, 0) \propto\left(H-H_{c}\right)^{\nu}
$$

We found that by adopting $f$ as a third order polynomial, the data around the critical point can be aligned along the scaling curve as shown in Fig.10. The fitting gives the value of the critical exponent of the conductivity $\nu=$ $1.6 \pm 0.2$.

The exponents for temperature and the magnetic field are uncommon to those reported so far in MIT's. This is not so surprising, however, considering that both the electron correlation and the disorder are important in the 
present MIT. In such cases, it is known that even the choice of the parameter to approach to the critical point changes the universality class of the system ${ }^{25)}$. It should be noted that the value of $\nu$ is close to the results of numerical scaling in non-interacting systems ${ }^{26)}$.

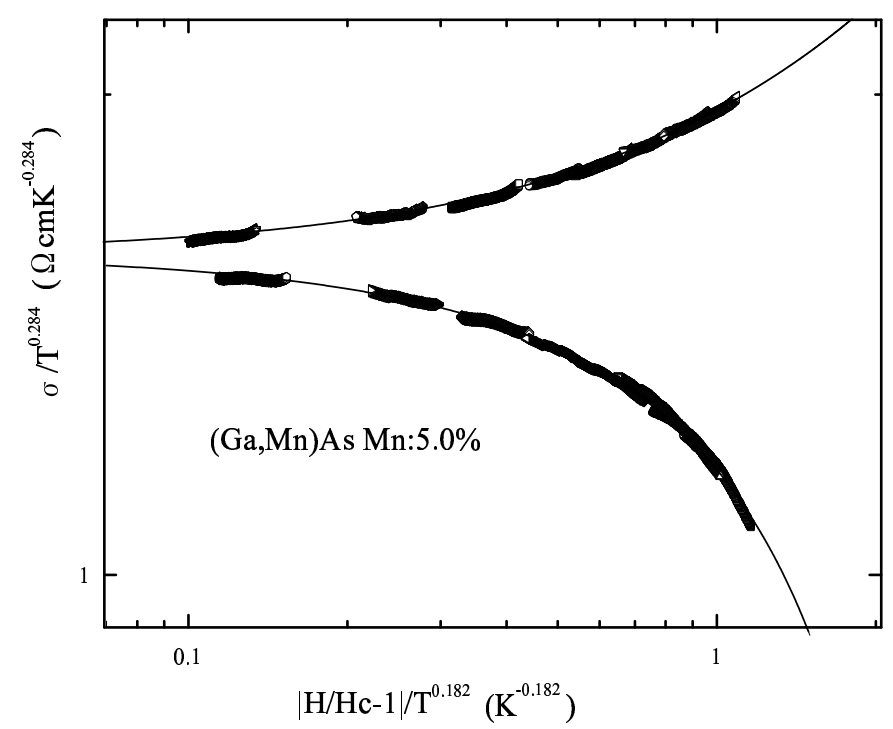

Figure 9: Two parameter scaling plot of the data shown in Fig.8. The formula is shown in the text.

It is hardly believed that the Coulomb repulsion is irrelevant in the present system. However the external field is considered to vary almost purely the degree of disorder because the effect of the external field is negligible besides ordering of random magnetic moments. And that would be the reason we got the value close to the result in noninteracting systems ${ }^{27)}$.

This work is partly supported by Grant-in-Aid for Scientific Research on the Priority Area "Spin Controlled Semiconductor Nanostructures" from the Ministry of Education, Science, Sports and Culture, Japan.

\section{REFERENCES}

1) H. Munekata, H. Ohno, S. von Molnár, A. Segmüller, L. L. Chang and L. Esaki, Phys. Rev. Lett. 63, 1849 (1989).

2) H. Ohno, A. Shen, F. Matsukura, A. Oiwa, A. Endo, S. Katsumoto and Y. Iye, Appl. Phys. Lett. 69, 363 (1996).

3) S. Koshihara, A. Oiwa, M. Hirasawa, S. Katsumoto, Y. Iye, C. Urano, H. Takagi and H. Munekata, Phys. Rev. Lett. 78, 4617 (1997).

4) Y. Satoh, D. Okazawa and J. Yoshino, to be published in Physica E.

5) A. Haury, A. Wasiela, A. Arnoult, J. Cibert, S. Tatarenko, T. Dietl and Y. Merle d'Aubigné, Phys. Rev. Lett. 79, 511 (1997).
6) F. Matsukura, H. Ohno, A. Shen and Y. Sugawara, Phys. Rev. B57, R2037 (1997).

7) K. Nishizawa, S. Suzuki and O. Sakai, PhysicaB, in press.

8) H. Akai, Phys. Rev. Lett. 81, 3002 (1998).

9) J. Okabayashi, A. Kimura, T. Mizokawa, A. Fujimori, T. Hayashi and M. Tanaka, Phys. Rev. B58, R4211 (1998).

10) A. Oiwa, S. Katsumoto, A. Endo, M. Hirasawa, Y. Iye, H. Ohno, F. Matsukura, A. Shen and Y. Sugawara, Solid State Commun. 103, 209 (1997).

11) H. Shimizu, T. Hayashi, T. Nishinaga and M. Tanaka, Appl. Phys. Lett. 74, 398 (1999).

12) In ref.15, they convered the surface with GaAs wafers in order to prevent the escape of As atoms.

13) A. Oiwa, A. Endo, S. Katsumoto, Y. Iye, H. Ohno and H. Munekata, Phys. Rev. B59, 5826 (1999)

14) J. De Boeck, R. Oesterholt, A. Van Esch, H. Bender, C. Bruynseraede, C. Van Hoof, and G. Borghs, Appl. Phys. Lett.68, 2744 (1996).

15) A. Van Esch, L. Van Bockstal, J. De Boeck, G. Verbanck, A. S. van Steenbergen, P. J. Wellmann, G. Grietens, R. Bogaerts, F. Herlack and G. Borghs, Phys. Rev. B56, 13103 (1997).

16) T. Hayashi, Y. Hashimoto, S. Katsumoto and Y. Iye, submitted to Appl. Phys. Lett.

17) K. Hirakawa, S. Katsumoto, T. Hayashi, Y. Hashimoto and Y. Iye, unpublished.

18) M. E. Fisher and R. J. Burford, Phys. Rev. 156, 583 (1967).

19) A. J. Millis, P. B. Littlewood and B. I. Shraiman, Phys. Rev. Lett. 74, 5144 (1995).

20) Y. Ishiwata, M. Watanabe, R. Eguchi, T. Takeuchi, Y. Harada, S. Shin, T. Hayashi, Y. Hashimoto, S. Katsumoto and Y. Iye, submitted to Phys. Rev. Lett.

21) G. van der Laan and I. W. Kirkman, J. Phys.: Condens. Matter 4, 4189 (1992).

22) R. Shioda, K. Ando, T. Hayashi, and M. Tanaka, Phys. Rev. B58, 1100 (1998).

23) N. F. Mott, "Metal-Insulator Transitions" (2nd ed., Taylor and Francis, 1990).

24) D. Belitz and T. R. Kirkpatrick, Rev. Mod. Phys. 66, 261 (1994).

25) M. Imada, Fujimori and Y. Tokura, Rev. Mod. Phys. 70, 1039 (1998).

26) T. Kawarabayashi, B. Kramer and T. Ohtsuki, Phys. Rev. B57, 11842 (1998).

27) We thank K. M. Ito for this comment. 